\title{
Tratamiento de lesiones de caries interproximales mediante el uso de infiltrantes
}

\author{
Treatment of approximal caries lesions using infiltrants
}

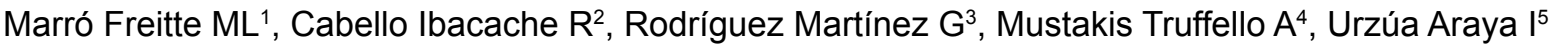

\section{RESUMEN}

Basados en el respeto por la estructura dentaria junto a un adecuado tratamiento y detención de las lesiones de caries, se ha desarrollado ICON $®$, un sistema que penetra los tejidos afectados con una resina de baja viscosidad fotopolimerizable (infiltrante) sin necesidad de la apertura mecánica de una cavidad. De este modo se introducen los "infiltrantes" como herramientas para el tratamiento de las lesiones proximales. El "infiltrante" actúa bloqueando la progresión de la lesión de caries y estabilizando la estructura dentaria. En este reporte se muestra la técnica de infiltración con el uso del kit ICON®, mediante la presentación de un caso clínico de lesión proximal extendida al tercio externo de la dentina.

Rev. Clin. Periodoncia Implantol. Rehabil. Oral Vol. 4(3); 134-137, 2011.

Palabras clave: Lesiones proximales de caries, infiltrantes, sellantes.

\section{ABSTRACT}

Based on the respect for the tooth structure with adequate treatment and arrest of carious lesions, ICON $®$ has been developed. This system enters the affected tissue with a low-viscosity light-curing resin (infiltrant) with no need for mechanical opening of a cavity. This will introduce the infiltrants as tools for the treatment of proximal lesions. The "infiltrant" works by blocking the progression of caries lesion and stabilizing tooth structure. This report shows the technique with the use of the ICON $®$ kit, by presenting a case of proximal carious lesion extended to the outer third of dentin.

Rev. Clin. Periodoncia Implantol. Rehabil. Oral Vol. 4(3); 134-137, 2011.

Key words: Approximal caries, infiltrant, sealants.

\section{INTRODUCCIÓN}

La lesión de caries se puede definir como una disolución química de los tejidos duros del diente por ácidos de origen bacteriano, producto de la degradación de azúcares de bajo peso molecular ${ }^{(1)}$. En etapas iniciales de la lesión cariosa, la disolución de sus constituyentes (PO4, $\mathrm{Ca}$ y $\mathrm{OH}$ ) se produce a partir de la capa subsuperficial y cuerpo de la lesión, con una pérdida de mineral de hasta $30-50 \%$ y donde el volumen de poros excede el $5 \%$ extendiéndose en profundidad en el esmalte y hacia la dentina. En cambio la capa superficial se caracteriza por estar relativamente intacta, mineralizada, con un grosor de 20 a 50 $\mu \mathrm{m}$ y donde el volumen de porosidad no exceden el $1 \%{ }^{(1)}$.

Clínicamente la manifestación más temprana, debido al aumento de porosidad, es la mancha blanca. Esta se visualiza como un esmalte de apariencia opaca y puede estar con la superficie intacta o microcavitada. Radiográficamente las lesiones de caries son evaluadas en las superficies proximales mediante el uso de técnicas de aleta mordida. El propósito de este examen es detectar lesiones ocultas al examen clínico, como sucede en las superficies proximales. Adicionalmente la imagen radiográfica nos ayuda a estimar la profundidad de las lesiones $y$, por tanto, constituye un elemento necesario para la decisión de tratamiento( ${ }^{(2)}$.

La caries dental ha experimentado un marcado descenso en los países industrializados ${ }^{(1)}$ y en Chile la información epidemiológica reciente da cuenta de este fenómeno ${ }^{(3)}$, aun cuando no existen estudios nacionales disponibles respecto a la prevalencia de lesiones interproximales. Un reciente estudio de prevalencia realizado con radiografías bitewing ${ }^{(14)}$ en adolescentes de 12 años de edad muestra una prevalencia de $58.5 \%$ de lesiones proximales en molares y premolares, con un COPD para estas mismas superficies de $1.6 \pm 2.2$ (DS).

De acuerdo a esta tendencia, es que en los últimos años se ha producido un incremento en el desarrollo de nuevas técnicas y materiales para el ejercicio de la odontología con un criterio más conservador, lo que implica la implementación de técnicas de remineralización por sobre la restauración de las lesiones ${ }^{(4,5)}$. Sin embargo, se ha observado un amplio rango de decisiones de tratamiento para las lesiones de caries proximales $^{(6)}$. Estudios muestran que el $18 \%$ de los tratantes declara que las lesiones proximales confinadas al esmalte requerirán un tratamiento restaurador, mientras que el $62 \%$ declara que el tratamiento restaurador es necesario cuando la lesión ha alcanzado el tercio externo de la dentina ${ }^{(7,8)}$, suponiendo que a esa profundidad la lesión de caries estaría cavitada, condición necesaria para indicar el tratamiento restaurador.

El tratamiento restaurador de lesiones proximales implica en la mayoría de los casos una preparación cavitaria lo que provoca un deterioro estructural de la pieza dentaria por pérdida del rodete marginal ${ }^{(9)}$ y la radiografía bitewing no nos entrega información respecto a cavitación, ya que se ha demostrado de un $59 \%$ de las superficies de molares premanentes y más de un $70 \%$ de las superficies de molares temporales que presentan lesiones de caries que radiográficamente alcanzan el tercio externo de la dentina no están cavitadas ${ }^{(15)}$.

Basados en el respeto por la estructura dentaria junto a un adecuado tratamiento, detención de las lesiones de caries y la duda razonable que plantea la profundidad de las lesiones de caries proximales en una radiografía bitewing, respecto a su condición de cavitación, un grupo de científicos de la Charité en Berlín y la Universidad de Kiel de Alemania desarrollaron ICON®, un sistema que utiliza una resina de baja viscosidad fotopolimerizable denominada "infiltrante" debido a que mediante la "infiltración" penetra en los tejidos afectados sin necesidad de la apertura mecánica de una cavidad. De este modo se introducen los infiltrantes como herramientas para el tratamiento de las lesiones proximales. Este sistema está indicado para lesiones de caries proximales con una extensión máxima D1 ó R3 según criterio de detección radiográfico (radiolucidez hasta el tercio externo de la dentina) $)^{(1,2)}$, y lesiones de mancha blanca en caras libres vestibulares. El diente es preparado mediante la desmineralización con un gel de ácido clorhídrico $(\mathrm{HCl})$ al $15 \%$, luego el tejido duro perdido es sustituido por una resina infiltrante $(I C O N \otimes)$ que penetra sellando los poros formados, incluso a una profundidad de hasta $800 \mu \mathrm{m}$. Es decir, el "infiltrante"

1. Cirujano Dentista. Programa de Magíster en Ciencia Odontológicas. Facultad de Odontología, Universidad de Chile. Chile.

2. Magíster en Ciencias Odontológicas. Facultad de Odontología, Universidad de Chile. Chile.

3. Magíster en Ciencias Odontológicas. Facultad de Odontología, Universidad de Chile. Chile.

4. Magíster en Ciencias Odontológicas. Facultad de Odontología, Universidad de Chile. Chile.

5. Magíster en Ciencias Odontológicas. Departamento de Odontología Restauradora, Facultad de Odontología, Universidad de Chile. Chile.

Correspondencia autor: Iván Urzúa Araya. ivanurzua@yahoo.com. Trabajo recibido el 14/02/2011. Aprobado para su publicación el 28/09/2011. 
actuaría bloqueando la progresión de la lesión de caries y estabilizando la estructura dentaria ${ }^{(10,11,16)}$.

Así, el objetivo de este reporte es mostrar la técnica de infiltración con el uso del kit ICON®, mediante la presentación de un caso clínico de una lesión proximal que se extienden hasta el tercio externo de la dentina.

\section{CASO CLÍNICO Y TRATAMIENTO}

Se seleccionó un paciente de 17 años, sexo femenino, cuyo diagnóstico clínico-radiográfico (radiografía bite wing estandarizada) detectó lesiones de caries proximales estrictas hasta R3, asintomáticas (Figura 1).

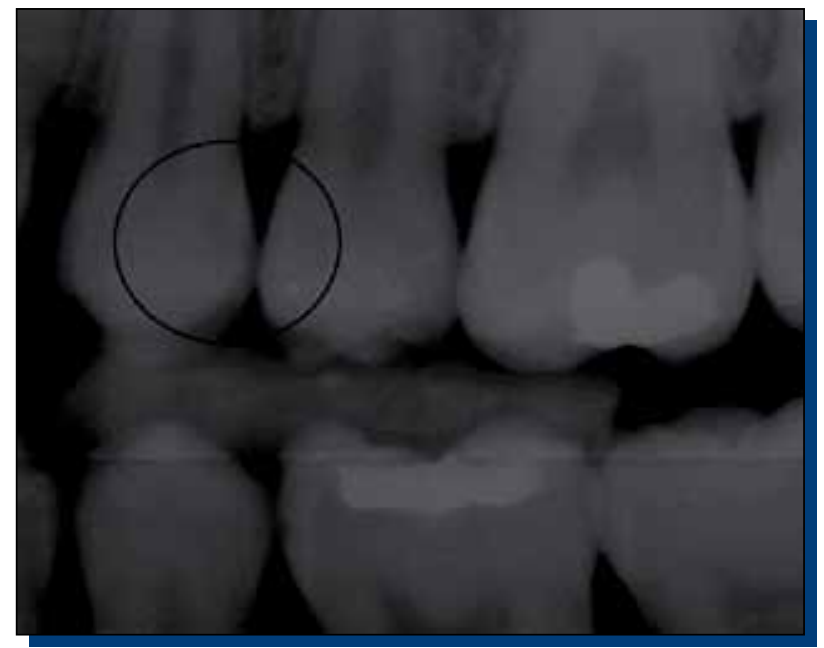

Figura 1. Pza. 12, lesión de caries distal en tercio externo de la dentina (R3).

Previo consentimiento informado del paciente, se realizó la técnica de separación temporal mediata de la pieza a tratar y su vecina, mediante elásticos de ortodoncia instalados en boca 24 horas antes de la aplicación de ICON $囚$, para así facilitar el diagnóstico de la lesión mediante examen visual y táctil como complemento al diagnóstico radiográfico (Figuras 2 y 3 ).

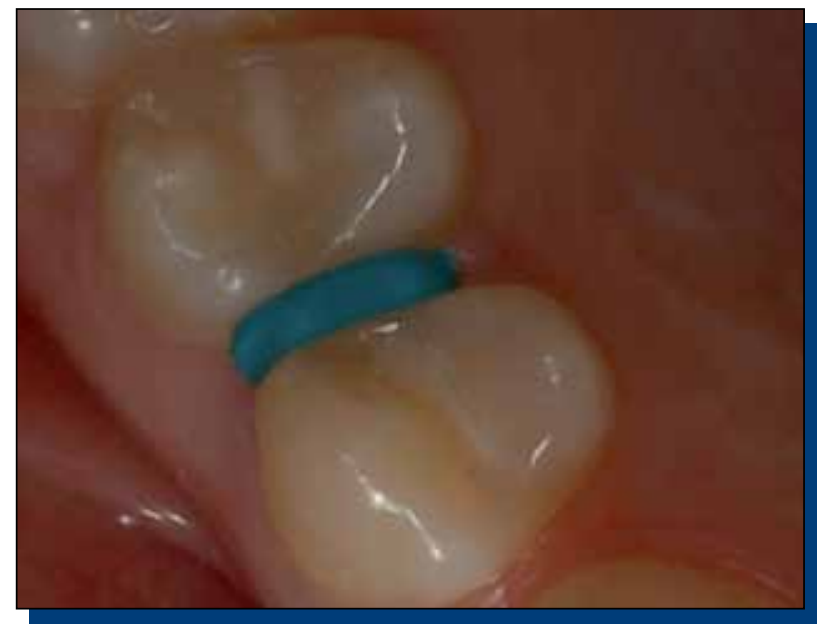

Figura 2.

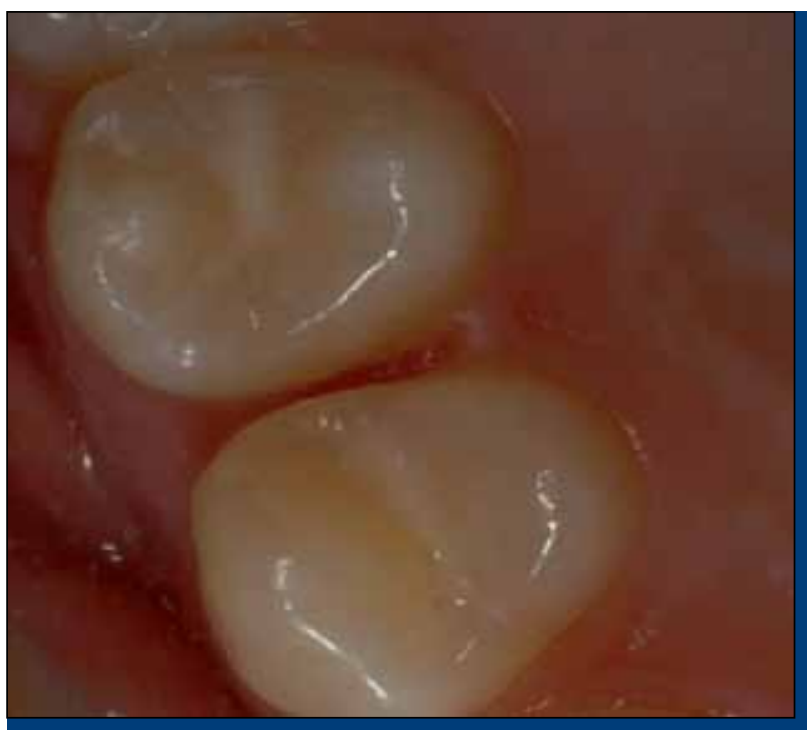

Figura 3.

Se trabajó con aislamiento absoluto de la hemiarcada de la pieza a tratar, previo al posicionamiento del clamps se aplicó anestesia tópica (Lidocaina $2 \%$, Mayon®) en la zona gingival. Se tuvo especial cuidado durante todo el proceso debido al tipo de ácido a utilizar y su potencial efecto sobre la mucosa (Figura 4).

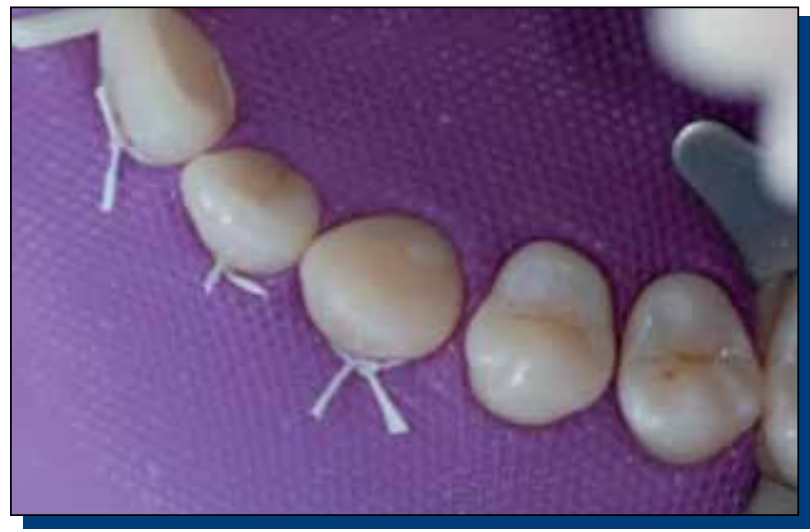

Figura 4.

Posteriormente se procedió a aplicar el infiltrante ICON $\circledast$, respetando las instrucciones y tiempos operatorios indicados por los fabricantes y dispuestas en el Kit.

\section{Instrucciones del Fabricante}

1. Separación interdental mediante cuña $I C O N \otimes$, la que se ubicará en posición hasta el final del proceso (Figura 5).

2. Aplicación de ICON Acid Etch (gel de ácido clorhídrico al 15\%) manteniéndolo por 2 minutos, obsérvese la opacidad del esmalte tratado (Figura 6).

3. Lavado de superficie durante un minuto y secado con aire (Figura 7)

4. Aplicación de ICON Dry por 30 segundos y secado con aire (Figura 8).

5. Primera aplicación de Resina Infiltrante ICON® manteniendo en posición durante 3 minutos (Figura 9).

6. Fotopolimerización por 40 segundos abarcando caras libres (vestibular-lingual/palatina) y oclusal (Figura 10).

7. Segunda aplicación de Resina Infiltrante ICON®, manteniendo en posición durante 1 minuto (Figura 11).

8. Fotopolimerización con las mismas indicaciones del paso 6.

9. Retiro de cuña, aislamiento (Figura 12).

Obsérvese la erosión provocada sobre la goma dique luego de realizado el tratamiento.

Tiempo clínico promedio empleado: 30 minutos. 


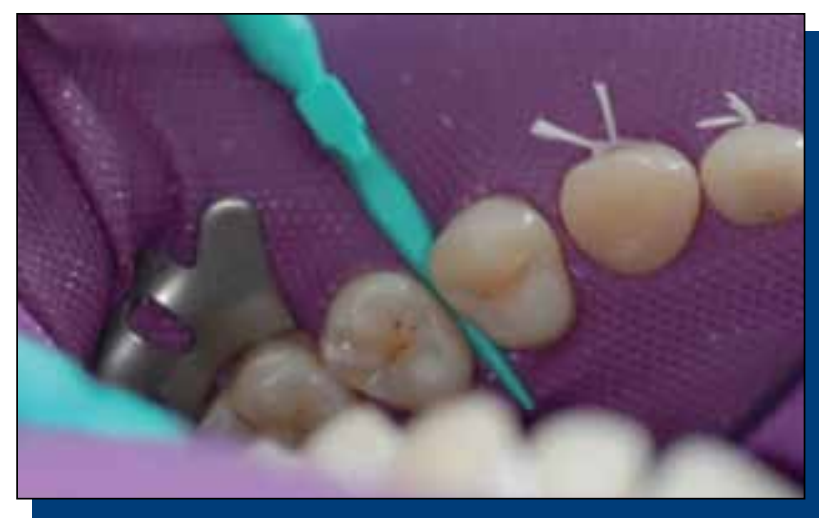

Figura 5.
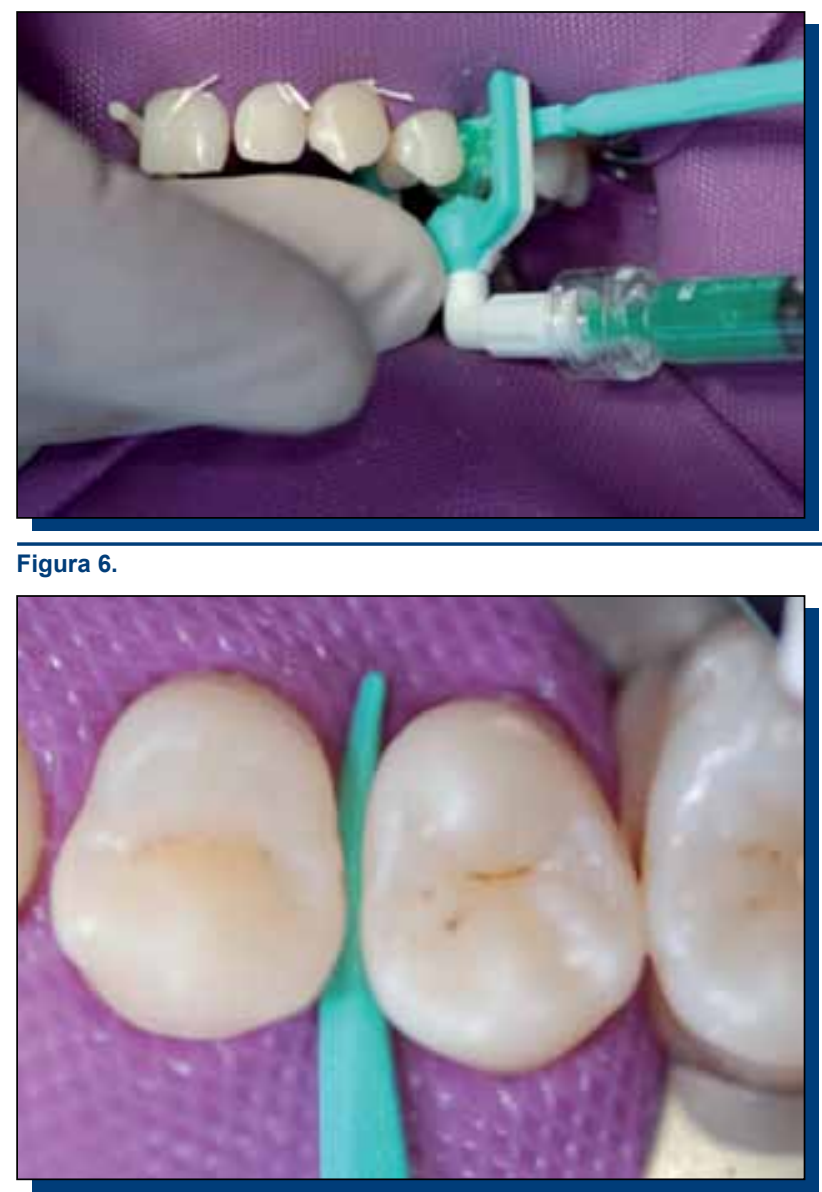

Figura 7.

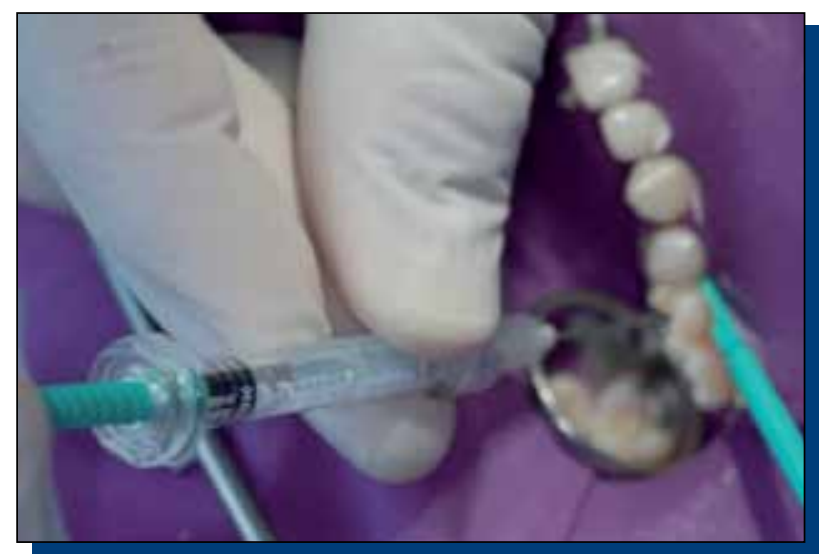

Figura 8.

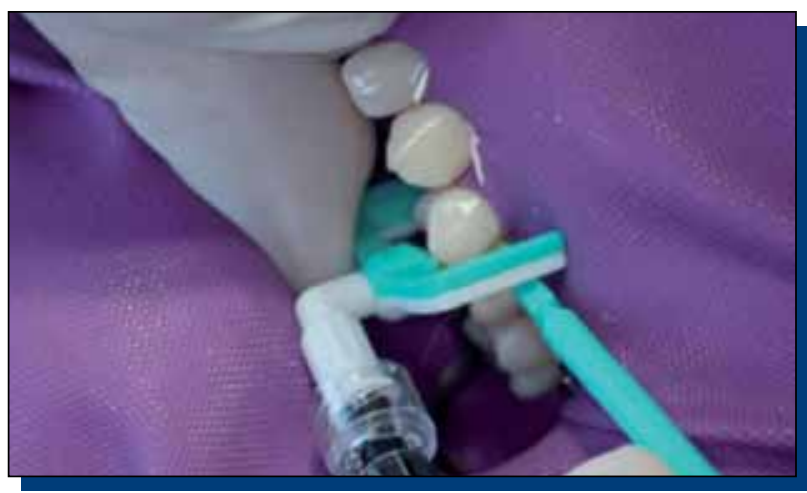

Figura 9.

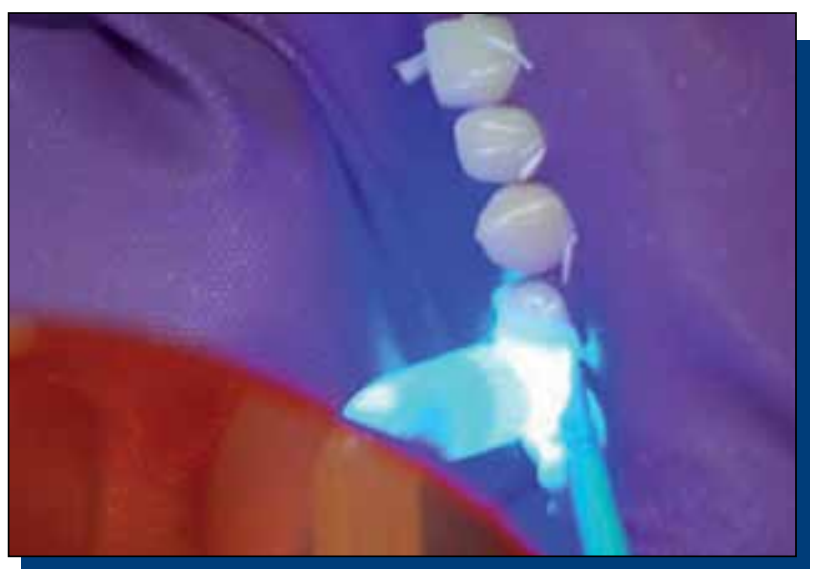

Figura 10.

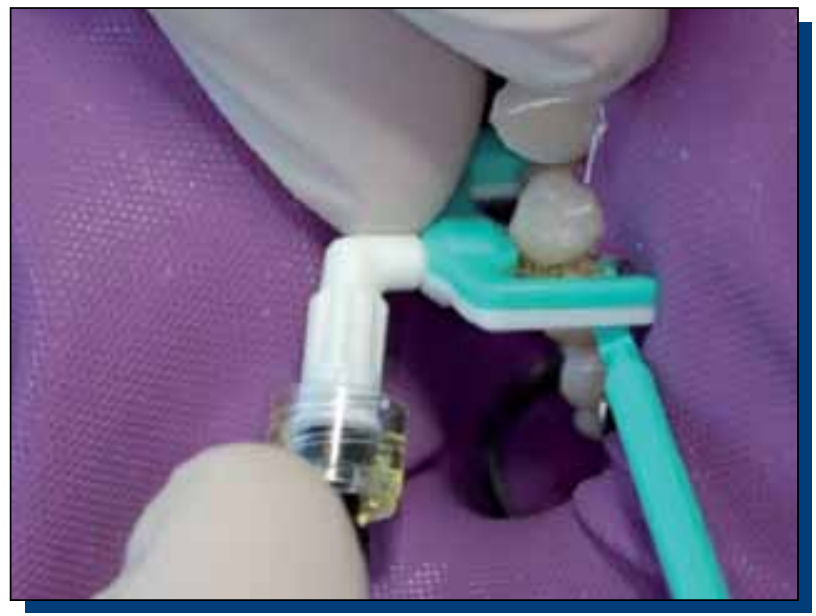

Figura 11.

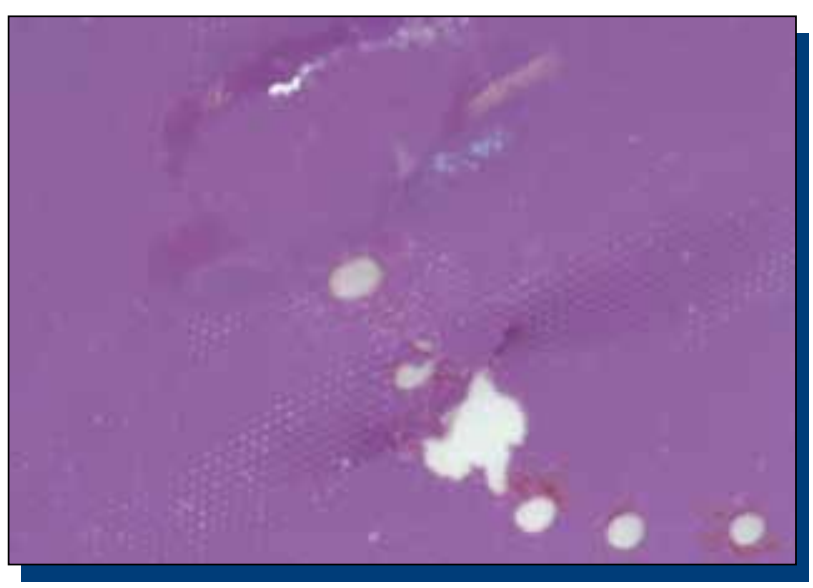

Figura 12. 
Al examen clínico inmediato, la pieza dentaria se observa con infiltrante translúcido, textura lisa perceptible al realizar el examen con sonda de caries, mucosa de aspecto normal (Figura 13).

La paciente relató no presentar molestias durante o después de la aplicación de ICON $®$

El control clínico fue realizado una semana después de la aplicación del infiltrante y la paciente relató no presentar molestias mediatas al tratamiento.

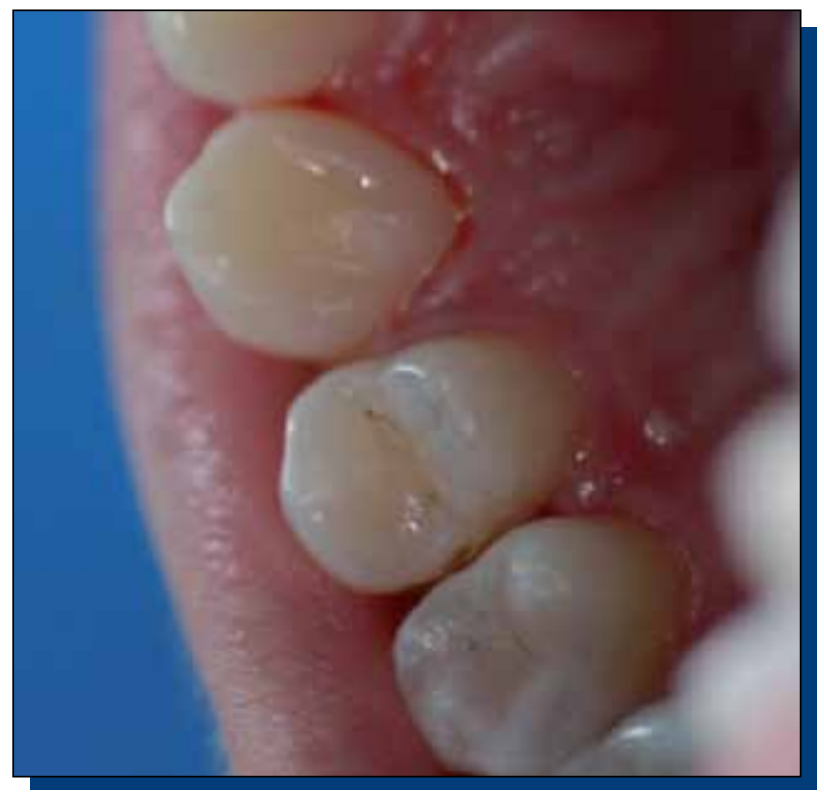

Figura 13

\section{DISCUSIÓN}

La infiltración del cuerpo de la lesión mediante una resina de baja viscosidad se puede ver obstaculizada por la capa superficial del esmalte la cual es una barrera debido a su bajo volumen de poros. La afirmación anterior se confirma en el estudio in vitro realizado por Paris $y$ cols. ${ }^{(10)}$ en el que fueron utilizados premolares y molares humanos extraídos, cuyo grupo control no fue grabado con ácido para luego ser infiltrados, el resultado fue la no penetración de la resina de baja viscosidad (infiltrante) en este grupo. Debido a lo anterior, para realizar esta técnica, es esencial la remoción o perforación de esta capa mediante el acondicionamiento con ácido.

Según el mismo estudio, la penetración de una resina es significativamente mayor si la pieza dentaria es preparada con un gel de $\mathrm{HCL}$ al $15 \%$ versus un gel de ácido fosfórico al $37 \%$, con una diferencia de profundidad de penetración de [58(SD 37) $\mu \mathrm{m}$ ] v/s [18(SD11) $\mu \mathrm{m}$ ] respectivamente ${ }^{(10)}$.

Al analizar el efecto de estos dos ácidos, esta vez en el contenido mineral de lesiones de esmalte incipientes de dientes primarios, se concluye que el tratamiento con $\mathrm{HCL}$ por 120 segundos lleva a una completa erosión de la capa superficial, podiendo tener una influencia positiva en la penetración de la resina infiltrante ${ }^{(11,12)}$.

Antes de decidir rehabilitar una pieza dentaria posterior, que presenta una lesión de caries proximal hasta el tercio externo de la dentina, utilizando el método operatorio tradicional (clase II de Black), debemos considerar diversos aspectos; principalmente estructurales, que nos podrían llevar a optar por otro método. El acceso a la lesión de caries que se realiza, generalmente, a través de la cara oclusal. Esto implica necesariamente la remoción de la cresta marginal, con la consecuente pérdida estructural (de $30 \%$ a $60 \%$ de la pieza dentaria natural)(8)

A la necesidad de una planificación previa al diseño de la cavidad, de manera tal que los márgenes de la restauración eviten áreas de alto estrés mecánico, se debe tener en cuenta además de la lesión de caries, factores tales como parafunciones, maloclusión, edad y hábitos de higiene. Si no existe esta planificación previa, el tratamiento restaurador puede fracasar debido a la posibilidad de fractura, tanto de la restauración como de la pieza dentaria, que llevará a infiltración y el desarrollo de lesiones de caries secundaria.

El potencial daño de las superficies proximales vecinas al realizar una intervención operatoria, sin la utilización de protección adecuada, es otro problema al restaurar lesiones proximales. Qvist y cols. en 1992, en su estudio sobre progresión de caries proximales en relación al daño de preparaciones iatrogénicas, muestra que en el $70 \%$ de preparaciones clase II el diente adyacente es dañado(13).

Finalmente, al realizar una restauración operatoria, inevitablemente el diente entrará en el "ciclo restaurativo"(13) que, con el paso de los años, involucrará cada vez mayor pérdida de tejido dentario remanente y necesidad de tratamientos cada vez más invasivos.

El uso de resina infiltrante ICON ${ }^{8}$ es una buena alternativa de tratamiento microinvasivo, el cual logra controlar el proceso de caries preservando la estructura dental sin que el diente entre en el "ciclo restaurativo", respetando así las estructuras dentarias vecinas.

\section{CONCLUSIONES}

La técnica con infiltrante es menos invasiva que el tratamiento operatorio tradicional, basado en la restauración de preparaciones cavitarias clase II de Black. El uso de infiltrantes constituye un procedimiento clínico de menor complejidad que los tratamientos disponibles actualmente y es mejor aceptado por el paciente, dado que no requiere de técnica anestésica.

\section{CONFLICTOS DE INTERÉS}

Los autores declaran no tener conflictos de interés.

\section{REFERENCIAS BIBLIOGRÁFICAS}

1. Fejerskov O, Kidd E, editors. Dental Caries: The Disease and its Clinical Management. Blackwell Munksgaard Second Edition, 2008; 209-230.

2. Pitts NB, Kidd E. Some of the factors to be considered in the prescription and timing of Bite Wing radiography in the diagnosis and management of dental caries. J Dent, 1992; 20: 74-84.

3. Soto L, Tapia R, Jara G. Diagnóstico Nacional de salud Bucal del Adolescente de 12 años y Evaluación del Grado de Cumplimiento de los Objetivos sanitarios de Salud Bucal 2000-2010. www.minsal.cl.

4. Eklund S, Pittman J, Smith R. Trends in dental care among insured Americans: 1980 to 1995. JADA, 1997; 128: 171-178.

5. Selwitz R, Ismail A, Pitts N. Dental Caries. Lancet, 2007; 369: 51-59.

6. Baratieri LN y cols. Procedimientos preventivos y restauradores. Quintessence Books. Sao Paulo; Primera edición 1993.

7. Mejare I. Bitewing examination to detect caries in children and adolescentswhen and how often? Dent Update, 2005; 32: 588-590,593-594.

8. Mejare I, Malmgren B. Clinical and radiographic appearance of proximal carious lesions at the time of operative treatment in young permanent teeth. Scand J Dent Res, 1986; 94: 19-26.

9. Barrancos Mooney. Operatoria Dental. Edit. Med. Panamericana 2006.
10. Meyer-Lückel $\mathrm{H}$, Paris $\mathrm{S}$. Micro-invasive caries treatment with resin infiltration. www.holtdentalsupply.com

11. Paris S, Meyer-Lueckel $\mathrm{H}$, Kielbassa AM. Resin infiltration of natural caries lesions. J Dent Res, 2007; 86(7): 662-666.

12. Phark JH, Duarte S, Meyer Lueckel H, Paris S. Caries infiltration with resins: A novel treatment option for interproximal caries. Compend Contin Educ Dent, 2009 Oct; 30 Spec No 3: 13-17. Review.

13. Qvist V, Johannessen L, Bruun M. Progression of approximal caries in relation to iatrogenic preparation damage. J Dent Res, 1992; 71(7): 1370-1373.

14. Gowda S, Thomson WM, Foster Page LA, Croucher NA. What difference does use bitewing radiographs makes to epidemiological estimates of dental caries prevalence and severity in a young adolescent population with high caries experience? Caries Res, 2009; 43: 436-441.

15. Pitts NB, Rimmer PA. An in vivo comparison of radiographic and directly assessed clinical caries status of posterior approximal surfaces in primary and permanent teeth. Caries Res, 1992; 26: 146-152.

16. Paris S, Meyer-Lueckel H. Inhibición the caries progression by resin infiltration in situ. Caries Res, 2010; 44: 47. 